\title{
Comment évaluer un processus innovant? Cas de l'amélioration quantitative et qualitative de la fumure organique au champ
}

\author{
M. Blanchard ${ }^{1,2 *}$ M. Koutou ${ }^{2}$ E. Vall ${ }^{1,2}$ S. Bognini ${ }^{3}$
}

\begin{abstract}
Mots-clés
Fertilité du sol - Amendement organique - Engrais - Innovation Evaluation de l'impact ex post Afrique occidentale.
\end{abstract}

\begin{abstract}
Résumé
La fertilité des sols de l'Afrique de l'Ouest est soumise à de fortes pressions anthropiques, avec I'augmentation démographique, I'extension des surfaces cultivées et I'abandon de la pratique de la jachère. En mobilisant une démarche de recherche action en partenariat, le projet Fertipartenaires a proposé de coconcevoir des innovations sur l'usage de la fumure organique, selon trois étapes : augmenter la capacité de production de fumure organique en construisant des fosses, améliorer la qualité de la production et raisonner son application au champ. Comment évaluer un processus innovant composé de plusieurs phases, inscrit à des échelles spatiales et temporelles différentes ? Comment insérer cette évaluation dans une démarche participative ? Une méthode participative d'évaluation en trois étapes a été proposée : (1) évaluation de l'effet direct de l'innovation (bilan partiel), (2) analyse des conséquences indirectes économiques, sociologiques et écologiques de l'innovation, et (3) analyse de la dynamique de l'innovation (adoption, diffusion et redéfinition). Deux modèles de production de fumure organique ont été testés : une fosse fumière installée à la concession et une fosse à compost en bord-champ. L'analyse des effets directs montre que l'usage de compost est économiquement plus avantageux que celui du fumier aux coûts d'opportunités plus élevés. Cependant, les deux modèles de production restent complémentaires et présentent tous deux un solde monétaire positif. La méthode utilisée permet de reconnaitre les effets directs de l'innovation sur l'économie de production de fumure organique. Elle permet de lancer un dialogue avec les acteurs autour de l'illustration simplifiée des effets directs de l'innovation. Elle doit cependant être complétée par l'analyse des conséquences indirectes et de la dynamique de l'innovation pour considérer les dimensions non prise en compte par les bilans partiels.
\end{abstract}

\section{INTRODUCTION}

Dans les zones cotonnières de l'Afrique de l'Ouest, la problématique de la baisse de la fertilité des sols est mise en avant par la recherche agricole depuis une cinquantaine d'années. L'augmentation démographique, l'extension des surfaces cultivées et le développement de l'élevage ont provoqué une augmentation des

1. Cirad, UMR Selmet, Montpellier, France.

2. Cirdes, URPAN, Bobo-Dioulasso, Burkina Faso.

3. UPPC-Tuy, Houndé, Burkina Faso.

* Auteur pour la correspondance

Tél. : 0661005872

E-mail : melanie.blanchard@cirad.fr pressions anthropiques sur les ressources naturelles qui remettent en cause les processus reconnus d'entretien, voire de restauration de la fertilité des sols, gage de durabilité des systèmes de production $(2,17)$. Les zones sylvopastorales se réduisent. Elles ne permettent plus les transferts de fertilité du saltus vers l'ager et la mise en jachère des terres agricoles est limitée (9). La recherchedéveloppement a conçu un ensemble de techniques favorisant l'intégration des activités agricoles et d'élevage en vue d'accroître la production de fumure organique (FO), de contribuer à l'entretien de la fertilité des sols et d'accroître les productions (14). Vulgarisées, ces techniques ont été partiellement adoptées par les paysans. Les incertitudes sur la durabilité des systèmes de production demeurent dans un contexte climatique, économique et institutionnel difficile, incertain et évolutif. 
Aujourd'hui, les producteurs de l'ouest du Burkina Faso disposent de peu d'infrastructures pour la production de fumure organique : seulement 30 p. 100 des exploitations de la province du Tuy possèdent une fosse fumière à la concession et 7 p. 100 une fosse à compost bord-champ (20). La production de FO s'appuie sur des techniques peu diversifiées, valorisant une faible fraction des ordures ménagères, des déjections animales et des résidus de culture produits sur l'exploitation. D'importantes pertes en biomasses sont observées car une partie des résidus de culture sont brûlés et une partie des déjections animales sont abandonnées sur les parcours (4). Les paysans ont une faible maîtrise des conditions de décomposition de ces biomasses (rapport $\mathrm{C} / \mathrm{N}$, aération, humidité ; 5). L'épandage de FO est souvent limité aux champs proches de la concession car les exploitations restent sous-équipées en moyen de transport. En effet, la moitié d'entre elles, seulement, possèdent une charrette et peuvent transporter le fumier sur les champs éloignés (20).

Face à cette situation, dans le cadre du projet Fertipartenaires (DCE, 2008-2012), nous avons coconçu avec les producteurs des innovations techniques, afin d'améliorer quantitativement et qualitativement la production de FO, et de raisonner son application au champ. Le processus innovant proposé se décline en trois composantes successives, s'inscrivant à des échelles temporelles et spatiales spécifiques :

- augmenter la capacité de production de FO en installant une fosse fumière à la concession * ou une fosse à compost bord-champ ;

- améliorer la qualité de la FO produite en prenant en compte les contraintes de transport des exploitations et en valorisant mieux les résidus de culture et les déjections animales disponibles dans les exploitations ;

- raisonner son application au champ.

Pour chacune de ces composantes, des modalités techniques ont été expérimentées en mobilisant une démarche de « recherche action en partenariat » (RAP) avec les paysans de la province du Tuy (ouest du Burkina Faso). Dans cette démarche, les acteurs sont impliqués à chaque étape de la recherche, depuis le diagnostic, la recherche de solutions, leur mise en œuvre et leur évaluation (7). Le projet a ainsi soutenu l'installation de 613 fosses fumières et 665 fosses à compost, réalisé 99 études des pratiques paysannes de production de FO et suivi 27 essais d'application raisonnée de FO au champ. L'objectif de cette étude a été d'identifier la méthode à utiliser pour évaluer, de manière participative, un processus innovant, construit avec les producteurs autour de plusieurs composantes.

L'évaluation peut être définie comme étant la collecte d'informations sur les caractéristiques et les résultats d'une action, afin d'élaborer un jugement objectif sur cette action et de lui donner une valeur selon des critères déterminés, afin d'améliorer son efficacité et de fournir des informations pour aider à la décision. Les méthodes d'évaluation divergent selon ses objectifs (16), les étapes du processus de recherche au sein desquelles elles sont utilisées (13), l'échelle de l'analyse (parcelle, exploitation, territoire, pays) et la nature des indicateurs retenus (22). Il est donc nécessaire de définir l'objectif de l'évaluation et le périmètre du système à évaluer sur lequel a eu lieu l'action.

Dans le cas particulier d'une innovation, l'évaluation vise à fournir des informations pour prévoir (évaluation ex ante) et diagnostiquer (évaluation ex post). Elle constitue un outil de pilotage et d'aide à la décision en matière notamment de changement de pratiques à encourager, d'investissement à privilégier, d'organisation sociale

* Concession : espaces et bâtiments à l'usage d'habitation, de stockage, de stabulation des animaux (bœufs de trait, vaches laitières, petits ruminants et volailles) et correspondant au siège de l'exploitation. à promouvoir. Afin de prendre en compte les résultats technicoéconomiques, les perceptions des acteurs et de comprendre les processus en cours de conception, Hall et coll. (11) recommandent d'adopter des méthodes mixtes quantitatives, produisant des indicateurs chiffrés, et qualitatives, aux résultats non quantifiables.

Il s'agit ici d'évaluer l'impact direct de l'innovation sur l'économie de la production de FO, leurs conséquences indirectes sur l'économie, la société et l'environnement. Il s'agit aussi d'appréhender leur dynamique locale (adoption, diffusion, redéfinition).

La première hypothèse a été que l'on pouvait réaliser une évaluation des effets directs en définissant, pour chaque composante de l'innovation, le périmètre du système à évaluer et en comparant la pratique innovante à une pratique de référence. La seconde hypothèse a été que l'évaluation des conséquences indirectes de l'innovation sur l'économie, la société et l'environnement nécessitait une approche qualitative mais aussi participative. Selon la troisième hypothèse, il était nécessaire, pour évaluer la dynamique d'une innovation, d'enquêter sur des exploitations n'ayant pas participé à l'expérimentation (adoption spontanée) et sur le suivi des exploitations ayant participé à la démarche de RAP (expérimentation puis adoption ou redéfinition).

Dans la première partie, les différentes composantes du processus innovant relatif à l'usage de la FO ont été présentées. Dans la deuxième partie, la méthode proposée pour l'évaluation participative des impacts de l'innovation a été développée. Enfin, dans la troisième partie, cette méthode d'analyse a été appliquée aux résultats de l'expérimentation d'usage de la FO.

\section{PROCESSUS INNOVANT EXPERIMENTE}

\section{Démarche de coconception de l'innovation}

La démarche de coconception de l'innovation retenue s'est appuyée sur un dispositif de gouvernance gérant le partenariat entre producteurs et chercheurs : le cadre de concertation villageois (CCV) qui est construit autour de groupements de producteurs volontaires pour faire face à une problématique partagée (14). La démarche s'est appuyée sur les quatre étapes de la démarche de RAP :

- des études diagnostics pour définir la problématique que souhaitent traiter les producteurs ;

- une session de formation des acteurs et/ou un voyage d'étude sur la FO visant à explorer les voies d'améliorations possibles. Les connaissances scientifiques et les savoirs locaux sur la FO sont alors hybridés et aboutissent à la définition du protocole de l'expérimentation ;

- un cahier des charges qui recueille les engagements des acteurs pour l'expérimentation servant à la contractualisation entre chercheurs, CCV et expérimentateurs. Les expérimentations sont alors mises en œuvre ;

- une évaluation participative des options expérimentées réalisée sur les trois composantes de l'innovation étudiée, soit la construction de fosses, la production et l'utilisation de FO.

\section{Composantes $d u$ processus innovant}

Les modalités expérimentées par les producteurs sur l'usage de la FO représentent une alternative aux pratiques actuelles. Elles se fondent sur quelques grands principes :

- un modèle simple de deux fosses par exploitation avec une fosse à compost bord-champ et une fosse fumière à la concession ;

- une démarche de RAP qui vise une amélioration progressive des pratiques actuelles (processus itératif et innovation incrémentale); 
- un grand nombre de producteurs expérimentent la construction de fosses, la production et l'utilisation de FO afin de comprendre les contraintes à l'adoption de l'innovation pour une diversité d'exploitations.

La figure 1 illustre l'organisation des composantes du processus innovant expérimenté.

Un processus innovant correspond au processus au cours duquel un nouveau concept est adopté par un groupe social et induit un changement de pratique (1). La création de fosse à compost et fosse fumière engendre des changements de pratiques à différentes échelles spatiales et temporelles. Par exemple, une fosse peut être utilisée pendant cinq ans sans nécessiter de réparations majeures, la production de FO en fosse est un processus d'une année et l'utilisation de FO dans les champs s'évalue au cours de la saison culturale suivante. Pour que l'évaluation prenne en compte cette différence de temporalité, les bilans partiels sont réalisés à l'échelle d'une fosse et sur un pas de temps annuel, échelle unique adoptée ici pour les comparaisons.

\section{Augmentation de la capacité de production et production améliorée de fumure organique}

L'augmentation de la capacité de production de FO est recherchée en construisant des fosses à compost dans les champs éloignés du village et des fosses fumières à la concession. Installer les lieux de production de FO là où les biomasses sont disponibles permet de réduire les temps de transport et de valoriser les déjections animales et les résidus de cultures jusque-là non valorisés. Les producteurs volontaires pour l'expérimentation s'engagent à creuser des fosses de $9 \mathrm{~m}^{3}$ et à les stabiliser avec du ciment fourni par le projet.

La fosse à compost bord-champ est remplie avec des tiges de cotonnier de l'année n-1 disponibles au champ avant la mise en culture (avril, année n). Elle ne nécessite que l'apport de deux charrettes de déjections animales pour amorcer la décomposition. L'humidification du mélange est assurée par l'eau de pluie. La

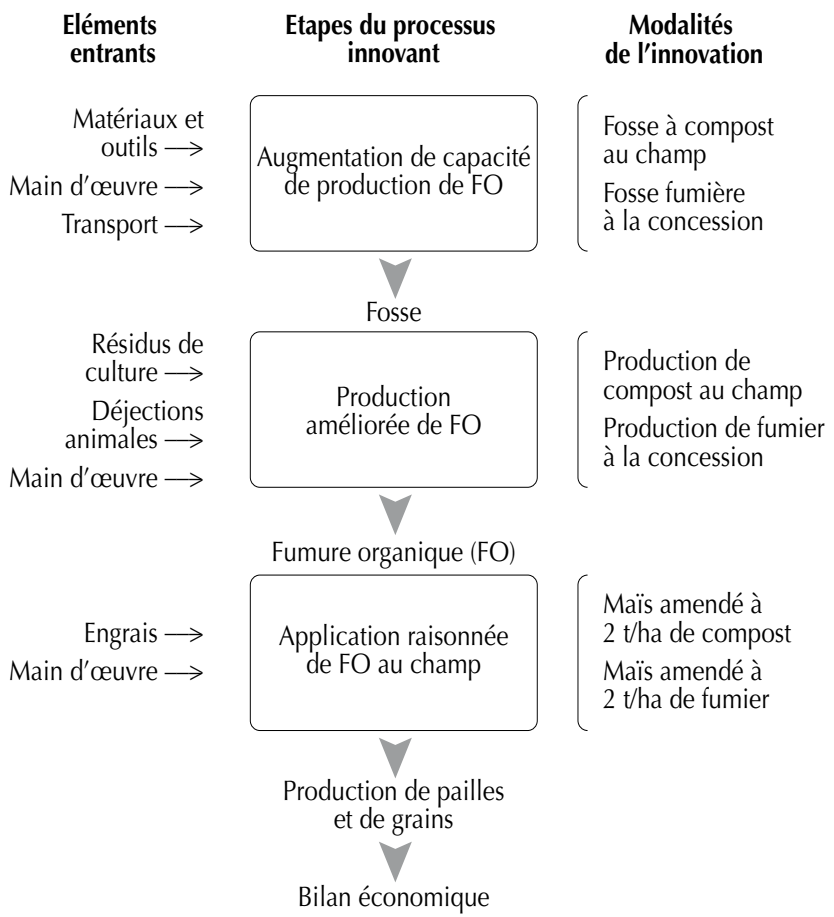

Figure 1 : production améliorée et application raisonnée de fumure organique. production du compost s'achève par son épandage avant le labour (année $n+1)$.

La fosse fumière à la concession est remplie progressivement avec les déjections des animaux, les refus de l'affouragement au fur et à mesure de leur disponibilité et les ordures ménagères (déchets organiques, cendres). La production s'achève par le transport du fumier au champ avant épandage et labour.

Les chercheurs et les producteurs ont formulé des propositions afin d'améliorer les pratiques de production de FO. Pour de meilleures conditions de décomposition, ils ont proposé d'ajouter aux résidus de récolte mis en fosse à compost un minimum de 20 p. 100 de déjections animales, afin d'équilibrer le rapport $\mathrm{C} / \mathrm{N}$, et ainsi de favoriser un compostage à chaud avec destruction des graines d'adventices et des germes. Le retournement du produit en fosse permettrait de réactiver la fermentation aérobie par les bactéries grâce à un apport d'oxygène (18). Enfin, l'arrosage du mélange au remplissage aurait déclenché la décomposition avant l'arrivée des pluies, mais cette modalité n'a pas été retenue par les producteurs qui ont estimé que la période de remplissage était déjà chargée en travaux agricoles. Des propositions d'amélioration supplémentaires ont été proposées à quelques paysans avec l'enrichissement des fosses au phosphate tricalcique (Burkina Phosphate ${ }^{\odot}, 25$ p. 100 de $\mathrm{P}_{2} \mathrm{O}_{5}$ ) ou l'ajout d'activateur de compost (Compost Plus ${ }^{\circledR}$ ).

\section{Application raisonnée de FO au champ}

La FO produite dans chaque type de fosse a été appliquée sur des parcelles de maïs. Sur des parcelles de 0,25 ha, une moitié « test » a reçu la dose recommandée de FO de $5 \mathrm{t}$ de matière sèche (MS) par hectare tous les deux ans (3), laissant la seconde moitié « témoin » non amendée. Les deux parties ont reçu la dose d'engrais minéraux préconisée par l'encadrement $(150 \mathrm{~kg} / \mathrm{ha}$ de NPK, $50 \mathrm{~kg} /$ ha d'urée).

\section{Caractéristiques de l'échantillon}

Les essais ont été conduits au cours de trois campagnes agricoles (2008-09, 2009-10 et 2010-11), dans sept villages et une commune rurale de la province du Tuy, représentative de la zone cotonnière de l'ouest du Burkina Faso. L'échantillon d'expérimentateurs pour chaque composante du processus innovant est présenté dans le tableau I : 75 p. 100 des producteurs expérimentateurs étaient des agriculteurs pratiquant l'agriculture et disposant d'animaux de trait ; 18 p. 100 étaient des agroéleveurs combinant l'agriculture et l'élevage, et seulement 7 p. 100 étaient des éleveurs cultivant des surfaces très réduites mais disposant de grands troupeaux (20).

\section{Tableau I}

Echantillon des producteurs expérimentateurs

\begin{tabular}{ll}
\hline Etapes du processus innovant & Echantillons \\
\hline $\begin{array}{l}\text { Augmentation de la capacité } \\
\text { de production de FO }\end{array}$ & $\begin{array}{l}\text { Bilan chez } 919 \text { volontaires } \\
(1684 \text { fosses })\end{array}$ \\
Amélioration de & Enquête auprès de \\
la qualité de FO & 99 volontaires \\
Application raisonnée & Suivi de 27 parcelles \\
de FO au champ & paysannes d'essai
\end{tabular}

FO : fumure organique 
METHODE D'EVALUATION D'UN PROCESSUS INNOVANT

\section{Démarche générale d'évaluation participative du processus innovant}

Une innovation technique ou organisationnelle, simple ou complexe, engendre des effets directs sur l'exploitation, des conséquences indirectes sur l'économie, la société et l'environnement, et une dynamique au sein de la communauté locale : adoptions spontanées par des agriculteurs n'ayant pas participé à l'expérimentation ou redéfinitions de l'innovation. La démarche d'évaluation des impacts de l'innovation proposée est présentée dans la figure 2.

\section{Evaluation des effets directs de l'innovation : de la préparation au bilan partiel}

La méthode du budget partiel représente un outil d'aide à la décision permettant d'analyser les effets de l'adoption d'une innovation sur le fonctionnement de l'exploitation $(6,10)$. Elle permet de traduire en terme monétaire les effets favorables ou défavorables d'un changement sur le fonctionnement de l'exploitation lié à l'adoption de l'innovation. Dans ce cas d'étude, nous avons choisi d'évaluer séparément chaque composante du processus innovant par le calcul de sous-bilans afin d'étudier la pertinence des différentes modalités proposées et de discuter des voies d'améliorations possibles. Afin d'évaluer l'ensemble du processus innovant, les trois composantes doivent être analysées sur un pas de temps identique (une année) et à la même échelle (une fosse). Une situation de référence doit être définie pour chaque composante pour servir de point de comparaison.

Les améliorations engendrées par l'adoption de l'innovation sont décrites par l'économie des charges réalisée (comme la réduction des doses d'engrais minéraux) et les produits nouveaux obtenus (gains en paille, grain). Les effets défavorables engendrés par l'innovation sont exprimés à travers l'augmentation des charges (comme le transport, le travail supplémentaire) et la perte d'usage de certaines ressources (comme la réduction du stock de fourrage par compostage de pailles). La différence entre les termes d'amélioration et de détérioration constitue le solde du bilan partiel. Le tableau II présente les charges et les produits pris en compte dans l'établissement des budgets partiels pour chaque composante du processus innovant.

L'analyse des bilans partiels est ensuite enrichie par les remarques des producteurs permettant la prise en compte de considérations ne pouvant être monétarisées, donc non prises en compte par le bilan partiel. Ces éléments peuvent apporter une amélioration des bilans par un changement technique ou organisationnel intra-exploitation (meilleure organisation du travail, développement de compétence en maçonnerie, etc.) ou en termes de règles collectives (date d'ouverture de la vaine pâture, divagation, etc.). L'objectif de cette première étape a été de fournir quelques éléments d'évaluation et de disposer d'une représentation des effets directs de l'innovation. La présentation des bilans partiels aux producteurs expérimentateurs a permis de discuter des facteurs de variation des bilans et de construire des voies d'améliorations pour les différentes modalités testées. Les conditions préalables à la mise en place des innovations (moyens à mobiliser, objectifs des exploitations, etc.) ont également été identifiées.

\section{Evaluation des effets indirects}

Les conséquences indirectes du processus innovant sur l'économie, la société et l'environnement ont été définies par les chercheurs et les producteurs à l'aide d'une grille d'analyse commune (tableau III). Pour chacun des critères, les indicateurs proposés par les producteurs ont été explicités puis complétés par des indicateurs proposés par les chercheurs.

\section{Analyse de la dynamique du processus innovant}

Après l'expérimentation, la dynamique du processus innovant a été appréhendée dans les villages où la RAP a été menée. Des

Tableau II

Variables utilisées pour les bilans partiels

Economies des charges ou

génération de produits nouveaux

Augmentation de la capacité de production de fumure organique

\section{Augmentation des charges ou perte d'usage de ressources}

Main d'œuvre (creusement et stabilisation)

Matériaux utilisés (ciment, sable, gravillons, briques)

\section{Production améliorée de fumure organique}

Compostage au Compost produit

champ

Fumier à la

concession

Fumier produit
Amortissement de la fosse

Résidus de culture mobilisés non disponible pour le bétail Main d'œuvre (ramassage, remplissage, retournement)

Amortissement de la fosse

Main d'œuvre (ramassage, remplissage, retournement)

Poudrette de parc ajoutée aux pailles

Résidus de culture mobilisés non disponibles pour le bétail

Compost ou fumier produit

Transport de la FO

Main d'œuvre (épandage, désherbage supplémentaire) 


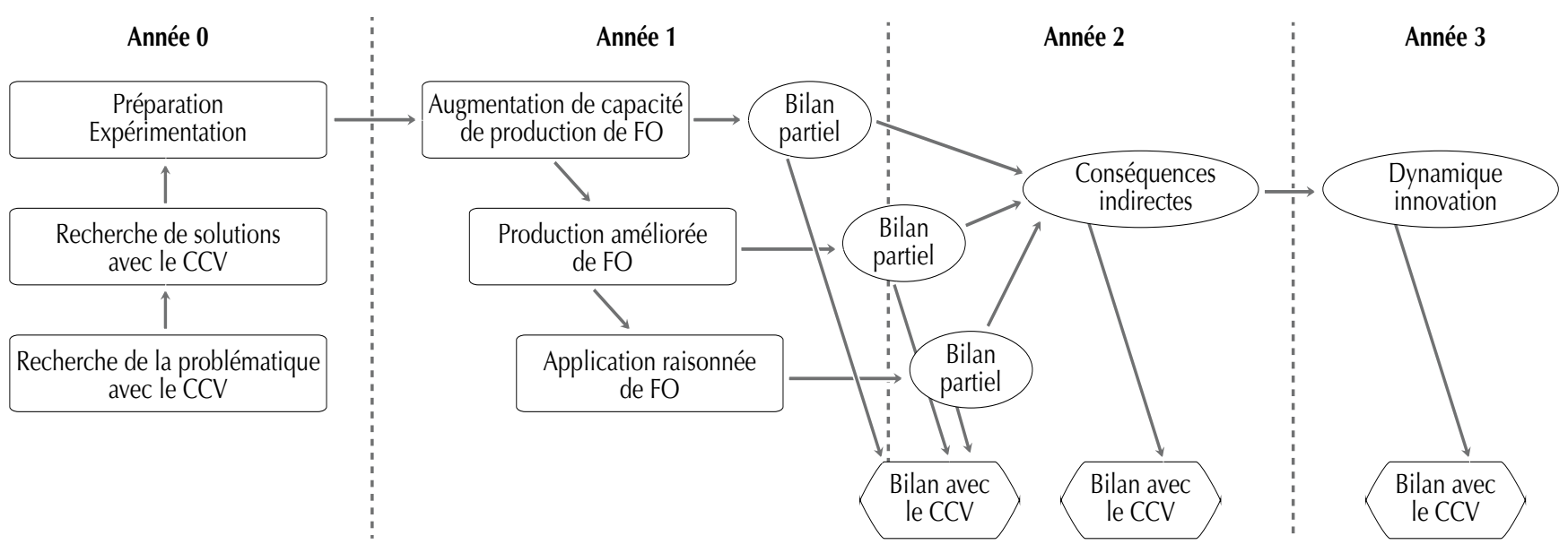

Figure 2 : méthode globale d'évaluation du processus innovant. CCV : cadre de concertation villageois ; FO : fumure organique.

\section{Tableau III}

Exemple de grille de caractérisation des conséquences indirectes de l'innovation

\begin{tabular}{|c|c|c|c|c|}
\hline \multicolumn{2}{|c|}{ Type de conséquences } & \multirow{2}{*}{$\begin{array}{l}\text { Capacité de production } \\
\text { de FO }\end{array}$} & \multirow{2}{*}{$\begin{array}{l}\text { Production améliorée } \\
\text { de FO } \\
\text { Commercialisation de FO }\end{array}$} & \multirow{2}{*}{$\begin{array}{l}\text { Utilisation raisonnée } \\
\text { de FO } \\
\text { Augmentation du revenu } \\
\text { agricole local }\end{array}$} \\
\hline Pôle économique & Revenu au niveau local & & & \\
\hline & $\begin{array}{l}\text { Travail (charge et } \\
\text { répartition) }\end{array}$ & $\begin{array}{l}\text { Charge et pénibilité du } \\
\text { travail de creusement }\end{array}$ & $\begin{array}{l}\text { Charge et pénibilité, } \\
\text { et période des travaux } \\
\text { de remplissage et de } \\
\text { retournement }\end{array}$ & $\begin{array}{l}\text { Charge et pénibilité, et } \\
\text { période des travaux de } \\
\text { vidange et d'épandage } \\
\text { Nb. de désherbages }\end{array}$ \\
\hline & $\begin{array}{l}\text { Sécurité alimentaire et } \\
\text { santé }\end{array}$ & $\begin{array}{l}\text { Risque de fosses inondées } \\
\text { et insalubrité }\end{array}$ & $\begin{array}{l}\text { Evacuation des déchets } \\
\text { ménagers et déjections sans } \\
\text { danger pour la santé humaine }\end{array}$ & $\begin{array}{l}\text { Production de céréales } \\
\text { additionnelle }\end{array}$ \\
\hline \multirow[t]{2}{*}{$\begin{array}{l}\text { Pôle humain } \\
\text { et social }\end{array}$} & $\begin{array}{l}\text { Formation } \\
\text { (connaissances), } \\
\text { maîtrise technique } \\
\text { et processus } \\
\text { d'apprentissage }\end{array}$ & $\begin{array}{l}\text { Compétence en } \\
\text { maçonnerie }\end{array}$ & $\begin{array}{l}\text { Connaissance du processus de } \\
\text { décomposition, des qualités } \\
\text { des FO }\end{array}$ & $\begin{array}{l}\text { Connaissance de la fertilité } \\
\text { des sols } \\
\text { Développement } \\
\text { d'indicateurs de fertilité } \\
\text { paysans }\end{array}$ \\
\hline & $\begin{array}{l}\text { Entraide (modalités, } \\
\text { développement) et } \\
\text { relations sociales } \\
\text { (travail, matériel, } \\
\text { échange technique) ou } \\
\text { concurrence (ressource, } \\
\text { savoir) }\end{array}$ & $\begin{array}{l}\text { Echange d'informations } \\
\text { (maçonnerie, type de sol) } \\
\text { Echange d'outils } \\
\text { Entraide (maçonnerie, } \\
\text { creusement) }\end{array}$ & $\begin{array}{l}\text { Echange d'expérience (suivi, } \\
\text { qualité des FO) } \\
\text { Entraide (remplissage, } \\
\text { retournement) } \\
\text { Echange (résidus, déjections) }\end{array}$ & $\begin{array}{l}\text { Echange d'expérience (effet } \\
\text { des FO sur sols, cultures, } \\
\text { dosage) } \\
\text { Entraide (vidange, transport) }\end{array}$ \\
\hline \multirow[t]{2}{*}{$\begin{array}{l}\text { Pôle } \\
\text { agroécologique }\end{array}$} & $\begin{array}{l}\text { Durabilité des } \\
\text { écosystèmes }\end{array}$ & & $\begin{array}{l}\text { Brûlis des résidus de culture } \\
\text { évité réduisant le risque de } \\
\text { feux de brousse }\end{array}$ & $\begin{array}{l}\text { Maintien de la biodiversité } \\
\text { (faune du sol) }\end{array}$ \\
\hline & Fertilité des sols & & $\begin{array}{l}\text { Quantité de FO disponible } \\
\text { Qualité de FO }\end{array}$ & $\begin{array}{l}\text { Rendement, enherbement, } \\
\text { taux de MOS } \\
\text { Développement } \\
\text { d'indicateurs de fertilité } \\
\text { paysans }\end{array}$ \\
\hline
\end{tabular}


enquêtes sur l'adoption de l'innovation ont été réalisées auprès d'exploitations n'ayant pas participé à l'expérimentation : adoption totale, partielle, déterminant de l'adoption. Des enquêtes sur la poursuite ou non de l'innovation ont été menées auprès des producteurs expérimentateurs : redéfinition des composantes, raisons de l'arrêt, perception des impacts, contraintes de la mise en œuvre.

\section{RESULTATS}

Cette partie illustre, à partir de références obtenues en Afrique de l'Ouest et des données de terrain, comment les effets directs du processus innovant ont été évalués par la méthode du bilan partiel et discutés avec les producteurs. Les bilans partiels de chaque composante du processus innovant sont présentés avant qu'un bilan à l'échelle de la gestion d'une fosse ne conclut la partie. Les résultats sur les conséquences indirectes et la dynamique locale de l'innovation ne sont pas présentés dans cet article.

\section{Augmentation de la capacité de production de la fumure organique}

L'augmentation de la capacité de production de FO des exploitations passe par le creusement de fosses et leur stabilisation au ciment. La situation de référence définie pour établir le budget partiel est la non-installation de fosse. Le budget partiel est présenté dans le tableau IV et les équations utilisées pour établir ce bilan sont détaillées dans l'encadré 1.

Ce bilan permet de calculer l'amortissement annuel de l'infrastructure à appliquer à l'ensemble du processus innovant proposé (2 $900 \mathrm{FCFA} /$ fosse, 4,4 €) ainsi que le montant de l'investissement de départ. L'amortissement est fonction de la durée de vie des fosses. D'après les déclarations des paysans, elle peut être estimée à cinq ans, sans réparations majeures. Elle peut atteindre 10 ans en cas de construction avec des briques de cuirasse et varie selon le mode de construction, le type de sol (taux d'argile, présence de cuirasse latéritique) et la maîtrise des techniques de maçonnerie.

La majorité des fosses creusées et stabilisées au ciment en Afrique de l'Ouest ont été construites avec du ciment subventionné par des programmes nationaux ou projets, ce qui allège l'amortissement annuel des infrastructures. Hors subvention, une fosse reviendrait à 5900 FCFA $(9 €)$ sur cinq ans. A titre indicatif, ce coût de revient équivaut à environ $0,4 \mathrm{sac}$ de $50 \mathrm{~kg}$ d'engrais composés NPKSB et trois sacs sont nécessaire pour fumer un hectare de maïs.

\section{Encadré 1}

\section{EQUATIONS UTILISÉES DANS LES BILANS PARTIELS}

Equation 1 : creusement et stabilisation $=$ nombre $d^{\prime} H_{. j}{ }^{*}$ prix H.j

H.j : homme jour. Nombre d'H.j estimé à 15 pour le creusement et 6 pour la stabilisation (projet Fertipartenaires). Prix H.j à 500 FCFA (déclaratif).

Equation 2 : ramassage des tiges, remplissage et retournement $=$ nombre $d^{\prime} H . j{ }^{*}$ prix $H . j$

Nombre $d^{\prime} H$.j estimé à 4 pour le ramassage des tiges, 4 pour le remplissage et 8 pour le retournement. Prix H.j à 500 FCFA (déclaratif).

Equation 3 : ramassage de la poudrette, fèces, pailles et retournement $=$ nombre $d^{\prime} H . j *$ prix $H . j$

Nombre d'H.j estimé à 6,5 pour le ramassage de la poudrette, 6,5 pour les fèces, 4,5 pour les pailles et 8 pour le retournement. Prix H.j à 500 FCFA (déclaratif).

Equation 4 : poudrette = quantité de fèces * prix des fèces Quantité de fèces estimée à $540 \mathrm{~kg}$ pour 2 bœufs en stabulation nocturne, 6 mois (Berger, 1996). Prix des fèces à 2500 FCFA par charrette de 125 kg (déclaratif).

Equation 5 : résidus mobilisés = quantité de pailles * prix des pailles

Quantité de pailles estimée à 540 kg (Berger, 1996). Prix des pailles à 10 FCFA/kg (déclaratif).

Equation 6 : épandage, désherbage supplémentaire $=$ nombre $d^{\prime} H . j / h a *$ prix H.j * surface fumée

Nombre d'H.j estimé à 10 pour l'épandage et 4,1 pour le désherbage (résult. pers.)

Surface fumée par 0,510 t de compost $=0,255$ ha (2 t/ha). Surface fumée par $0,833 \mathrm{t}$ de fumier $=0,417$ ha $(2 \mathrm{t} / \mathrm{ha})$

Equation 7 : transport de fumier = quantité de fumier / poids d'une charrette * prix transport

avec $833 \mathrm{~kg}$ de fumier produit à 300 FCFA la charrette de $150 \mathrm{~kg}$ de fumier (déclaratif).

Equation 8 : production = gain de production * surface fumée * prix du maïs ou des pailles

Gain en grains et pailles de maïs estimé à $780 \mathrm{~kg} / \mathrm{ha}$ par apport de 2 t/ha de fumure organique (résult. pers.). Prix du maïs à 100 FCFA $/ k g$ (déclaratif).

\section{Tableau IV}

Bilan partiel de I'augmentation de la capacité de production de fumure organique

\section{Economies des charges \\ ou génération de produits nouveaux (FCFA *)}

$\begin{array}{ll} & \text { Creusement (éq. 1) ** } \\ & \text { Stabilisation (éq. 1) } \\ \text { Construction } & \text { Sable (1 brouette) } \\ \text { des fosses } & \text { Gravillons }\end{array}$

Charges anciennes $\quad 0$

Produits nouveaux $\quad 0$

\author{
Augmentation des charges
}

ou perte d'usage des ressources (FCFA)

Charges nouvelles

Perte d'usage de ressources
Bilan (FCFA)

7500

3000

2500

$1500-14500$ sur 5 ans

14500

-2900 par an

* $1000 \mathrm{FCFA}=1,52 € ; * *$ éq. : équation (cf. Encadré 1) 
Les producteurs qui ont construit leurs fosses avec des briques de cuirasse latéritique ont réalisé un investissement supplémentaire qui doit être justifié par une utilisation plus longue des fosses (coût estimé à $3000 \mathrm{FCFA} /$ fosse, 4,6 €). Le tableau $\mathrm{V}$ présente les calculs de l'amortissement des fosses hors subvention selon les durées de vie estimées par les producteurs et les modes de construction. La maîtrise des techniques de maçonnerie et l'utilisation effective du ciment à bonne dose pour la construction des fosses permettent de réduire le prix de revient, même dans le cas où le ciment n'est pas à la charge des producteurs.

L'ensemble des facteurs de variation ont été discutés avec les expérimentateurs et les conditions nécessaires améliorant la solidité des fosses ont été définies (organisation collective mobilisant un maçon compétent, travail collectif pour le creusement, utilisation de briques, importance d'utiliser le ciment donné par le projet pour la construction des fosses, etc.)

\section{Production améliorée de fumure organique}

\section{Cas de la fosse à compost en bord-champ}

Le compostage des tiges de cotonnier en fosse est un mode de production de FO additionnel qui entre peu en concurrence avec la pratique actuelle. Selon cette situation de référence, la fumure organique produite est une poudrette de parc et les tiges de coton sont brûlées. Les tiges de coton utilisées sont ramassées en février après la vaine pâture des feuilles vertes et des capsules (8). Une fosse à compost peut contenir jusqu'à $1700 \mathrm{~kg}$ de tiges de cotonnier, correspondant environ à 1 ha de culture. Au moment du remplissage (mai), les tiges sont disposées en couche alternées avec des déjections animales (deux charrettes), transportées depuis le lieu d'attache des animaux. Au cours du compostage, la perte due à la décomposition de la matière organique a été estimée à 70 p. 100 de la matière initiale par expérimentation (résultats non publiés) et la production moyenne de compost dans une fosse à $510 \mathrm{~kg}$ MS. Le bilan partiel de la production de compost est présenté dans tableau VI.

D'après le bilan partiel, le prix de revient du compost produit en fosse bord-champ (hors coût d'installation) est de 8600 FCFA/ fosse $(13,1 €)$. Sur le marché local, la charrette de $150 \mathrm{~kg}$ de poudrette se négocie à 2500 FCFA $(3,8 €)$, ce qui est comparable au coût estimé par le bilan partiel pour une charrette de compost (2 529 FCFA, 3,9 €).

Les travaux de retournement sont également consommateurs en main d'œuvre et influencent largement le bilan partiel

\section{Tableau V}

Amortissement des fosses construites

\begin{tabular}{|c|c|c|c|c|c|}
\hline \multicolumn{2}{|c|}{ Modalité de construction } & \multirow{2}{*}{$\begin{array}{c}\text { Durée de vie } \\
\text { des fosses (années) }\end{array}$} & \multirow{2}{*}{$\begin{array}{c}\text { Coût global } \\
\text { d'une fosse (FCFA) * }\end{array}$} & \multicolumn{2}{|c|}{ Amortissement annuel } \\
\hline & & & & $\begin{array}{l}\text { Global } \\
\text { (FCFA) }\end{array}$ & $\begin{array}{l}\text { Hors coût du } \\
\text { ciment (FCFA) }\end{array}$ \\
\hline \multirow[b]{2}{*}{$\begin{array}{l}\text { Au ciment } \\
\text { et crépi }\end{array}$} & $\begin{array}{l}\text { Maîtrise de la maçonnerie, utilisation } \\
\text { effective du ciment }\end{array}$ & 5 & 29500 & 5900 & 2900 \\
\hline & $\begin{array}{l}\text { Non maîtrise de la maçonnerie, } \\
\text { utilisation de peu de ciment }\end{array}$ & 2 & 29500 & 14750 & 7250 \\
\hline \multirow{2}{*}{$\begin{array}{l}\text { Avec brique } \\
\text { de cuirasse }\end{array}$} & Maîtrise de la maçonnerie & 10 & 32500 & 3250 & 1750 \\
\hline & $\begin{array}{l}\text { Non maîtrise de la maçonnerie, } \\
\text { utilisation de peu de ciment }\end{array}$ & 5 & 32500 & 6500 & 3500 \\
\hline
\end{tabular}

$* 1000 \mathrm{FCFA}=1,52 €$

\section{Tableau VI}

Bilan partiel de la production de compost de tiges de cotonnier en fosse

\section{Economies des charges ou génération de produits nouveaux (FCFA *)}

Production améliorée de fumure organique

$$
\text { Charges anciennes } \quad 0
$$

\section{Compost (510 kg de MS)}

Produits nouveaux 0
Bilan (FCFA) ou perte d'usage de ressources (FCFA)

2000

Transport 2 charrettes fèces 2000

Retournement (éq. 2) $\quad 4000$

Charges nouvelles $\quad 8600$

$-8600$ 
(4 000 FCFA, 6,1 €). Cependant, l'aération par le retournement du compost est nécessaire pour la bonne décomposition des éléments et pour relancer l'activité bactérienne (18). Il reste peu effectué par les paysans, même si certains reconnaissent l'effet néfaste pour les cultures d'un compost mal décomposé.

La proposition d'enrichir le compost avec du phosphate tricalcique permet d'obtenir un produit riche en phosphate et ainsi d'amender les terres carencées. L'application de phosphate tricalcique directement au sol avant labour n'a jamais rencontré de succès auprès des paysans qui trouvent le produit trop poudreux donc difficile à appliquer et dont l'effet n'est obtenu qu'après sa solubilisation après trois ans passés dans le sol. Le mélanger dans le compost lève la première préoccupation des paysans. Le coût du Burkina phosphate $^{\odot}(5000$ FCFA / $50 \mathrm{~kg}, 7,62 €)$ doit être compensé par une augmentation de la production en maïs cumulé sur les trois ans suivant l'application de la FO de $588 \mathrm{~kg} / \mathrm{ha}$ (en considérant un prix déclaratif du maïs de $100 \mathrm{FCFA} / \mathrm{kg}$ ).

L'ajout d'activateur de compost (Compost Plus ${ }^{\circledR}$ ) doit permettre une décomposition rapide du compost chez les paysans qui peuvent maintenir les conditions d'humidité et d'aération nécessaires à l'utilisation de ce produit. Le coût du produit (6 $000 \mathrm{FCFA} / \mathrm{sachet}, 9,15 €$ ) peut être compensé dans le cas du compostage de saison sèche pour les maraîchers qui souhaitent disposer de FO dès la fin de l'hivernage.

\section{Cas de la fosse fumière à la concession}

La production de fumier en fosse peut s'évaluer par rapport à la pratique actuelle où de la poudrette de parc est directement utilisée sans traitement préalable. Pour produire du fumier en fosse, les paysans ramassent des déjections animales déposées la nuit dans les parcs ou les lieux d'attache des animaux et les vident dans la fosse $(1,4 \mathrm{~kg}$ de fèces MS/bovin/nuit ; 3$)$. Nous prenons l'exemple d'un paysan possédant deux bœufs de trait stabulés la nuit près de $\checkmark$ la fosse. Afin d'équilibrer l'origine des éléments mis en fosse, le paysan y apporte également les refus de l'affouragement et de la litière (1 kg de litière/nuit). La figure 3 illustre, de manière synthétique, le remplissage d'une fosse fumière au cours de l'année et le temps passé en fosse par chaque couche. La production de fumier dans une fosse est estimée à $833 \mathrm{~kg}$ MS.

L'amélioration des pratiques de production de fumier entraîne un changement de qualité du fumier et une augmentation de la quantité produite par transformation de résidus de culture. Le bilan partiel de la production améliorée de fumier est présenté dans le tableau VII.

D'après ce bilan partiel, la production de fumier en fosse revient à $22450 \mathrm{FCFA} /$ fosse $(34,2 €)$. La charrette de fumier de $150 \mathrm{~kg}$ devrait alors se négocier à 4043 FCFA $(6,2 €)$, ce qui est plus élevé que le prix déclaré sur le marché local par les paysans (2 500 FCFA, soit $3,8 €$ ). Ces résultats sont à analyser avec prudence car la FO fait l'objet de peu d'échanges commerciaux et la qualité de la FO est encore peu prise en compte dans ces échanges. De plus, la rémunération du travail n'est pas équivalente entre la main d'œuvre extérieure (rémunération à la tâche ou à l'heure) et familiale (répartition des gains).

Le bilan partiel de la production de fumier est plus négatif que celui de la production de compost car il présente des coûts d'opportunité plus élevés. Le retournement des fosses reste consommateur en temps et certains producteurs ne l'effectuent pas, avec des conséquences sur la qualité des fumiers produits. Un autre terme de détérioration du bilan est la mobilisation de résidus qui ont une valeur marchande et ont d'autres usages (par exemple, les pailles de céréales pour affouragement). La production de fumier est concurrentielle des pratiques actuelles, mais elle permet d'obtenir une fumure de meilleure qualité et d'augmenter le volume produit par la valorisation de nouvelles biomasses. La qualité de la FO produite (fumier vs poudrette) pourrait être évaluée à partir de gains de production mais aussi par rapport aux gains de temps sur les travaux de désherbage, un fumier bien décomposé apportant moins de graines d'adventices qu'une poudrette de parc.

\begin{tabular}{|c|c|c|c|c|}
\hline & $\begin{array}{l}\text { Nb. de } \\
\text { jours }\end{array}$ & $\begin{array}{l}\text { Eléments apportés } \\
\text { dans la fosse }\end{array}$ & $\begin{array}{c}\% \text { de } \\
\text { décomposition }\end{array}$ & $\begin{array}{c}\text { Fumier } \\
\text { produit }(\mathrm{kg}\end{array}$ \\
\hline $\begin{array}{l}\text { Fumier de saison } \\
\text { sèche chaude }\end{array}$ & 90 & $\begin{array}{l}\text { Déjections } \\
\text { et litière }\end{array}$ & 82,5 & 256 \\
\hline $\begin{array}{l}\text { Fumier de saison } \\
\text { sèche froide }\end{array}$ & 90 & $\begin{array}{l}\text { Déjections } \\
\text { et litière }\end{array}$ & 65 & 281 \\
\hline $\begin{array}{c}\text { Fumier de la période } \\
\text { des récoltes }\end{array}$ & 90 & Déjections & 47,5 & 120 \\
\hline $\begin{array}{c}\text { Fumier } \\
\text { d'hivernage }\end{array}$ & 90 & Déjections & 30 & 76 \\
\hline
\end{tabular}

Figure 3 : remplissage de la fosse fumière au cours du temps.

\section{Tableau VII}

Bilan partiel de la production de fumier en fosse

\begin{tabular}{|c|c|c|}
\hline & \multicolumn{2}{|c|}{$\begin{array}{l}\text { Economies des charges } \\
\text { ou génération de produits nouveaux (FCFA* }\end{array}$} \\
\hline \multirow{4}{*}{$\begin{array}{l}\text { Production } \\
\text { améliorée } \\
\text { de fumure } \\
\text { organique }\end{array}$} & Ramassage poudrette (éq. 3) & 325 \\
\hline & Charges anciennes & \\
\hline & Fumier (833 kg de MS) & \\
\hline & Produits nouveaux & \\
\hline
\end{tabular}

Augmentation des charges

ou perte d'usage de ressources (FCFA)

Ramassage fèces (éq. 3) ** 3250

Transport pailles (éq. 3)

2250

Retournement (éq. 3)

Charges nouvelles

4000

9500

Poudrette (éq. 4)

10800

Résidus mobilisés (éq. 5)

5400

0

Perte d'usage de ressources
Bilan (FCFA)

$-22450$ 


\section{Utilisation raisonnée de fumure organique au champ}

Pour évaluer l'application de la FO sur une culture de maïs, une culture conventionnelle de maïs fertilisé uniquement avec des engrais minéraux a été définie comme situation de référence. L'apport de FO sur une parcelle de maïs nécessite d'avoir du compost ou du fumier, de le transporter au champ, de le disposer en tas et de l'épandre avant le labour. L'utilisation de FO sur une culture de maïs permet une amélioration de la production en grain et en paille.

Les soldes des bilans partiels de l'application de compost (tableau VIII) et de fumier (tableau IX) sont positifs. Le compost permet un gain partiel estimé à 20081 FCFA $(30,6 €)$ et le fumier un gain de 31173 FCFA (47,5 €). La production d'une fosse fumière permet d'amender une surface plus grande que celle d'une fosse à compost $(0,417$ ha contre $0,255 \mathrm{ha})$.

Le type et la qualité de la FO utilisée au champ est un facteur de variation du bilan (réduction du transport pour le compost, amélioration potentielle des gains pour les fumures bien décomposées, désherbage supplémentaire pour le fumier).

Certains paysans apportent la FO au champ au moment du ramassage des résidus de culture (avril). La FO reste alors exposée trois mois au soleil. Il y a un partage des coûts de transport mais une altération de la qualité de la FO (12). D'autres producteurs transportent la FO quand les travaux de labour sont prêts à être réalisés. La FO est alors enfouie immédiatement après l'épandage (investissement en travail supérieur mais qualité de la FO conservée).

Les travaux d'épandage de la FO représentent un coût important des bilans partiels et sont plus ou moins pénibles selon la disposition des tas de FO sur la parcelle. Le temps d'épandage augmente nettement pour les faibles densités de tas (moins de 100 tas/ha) et diminue nettement pour une densité supérieure. De plus, la disposition de gros tas de FO avant l'épandage entraîne un amendement localisé fort qui peut provoquer des brûlures de plants à la levée.

\section{Bilans partiels de la construction de la fosse à l'utilisation de la FO}

Les bilans partiels des deux types de FO depuis la construction des fosses jusqu'à l'utilisation de la FO sont positifs : 8 581FCFA $(13,1 €)$ pour une fosse à compost et $5823 \mathrm{FCFA}(8,9 €)$ pour une fosse fumière. Ceci correspond à un gain de $16825 \mathrm{FCFA} / \mathrm{t}$ de MS de compost produit et utilisé $(25,7 € / \mathrm{t})$ et $6990 \mathrm{FCFA} / \mathrm{t}$ de MS de fumier produit et utilisé $(10,7 € / t$; tableau $\mathrm{X})$. La production et l'utilisation de compost serait économiquement plus rentable comparée à celle du fumier.

\section{Tableau VIII}

Bilan partiel de l'utilisation de compost sur une culture de maïs

\begin{tabular}{|c|c|c|c|c|c|}
\hline & $\begin{array}{l}\text { Economies des charges } \\
\text { ou génération de produits nouveaux }\end{array}$ & & $\begin{array}{l}\text { Augmentation des charges } \\
\text { ou perte d'usage de ressour }\end{array}$ & & $\begin{array}{l}\text { Bilan } \\
\text { (FCFA) }\end{array}$ \\
\hline & & & Epandage (éq. 6) ** & 1275 & \\
\hline & & & Désherbage suppléé (éq. 6) & 523 & \\
\hline Application & Charges anciennes & 0 & Charges nouvelles & 1798 & 20081 \\
\hline $\begin{array}{l}\text { de fumure } \\
\text { organique }\end{array}$ & Gain de production de grains (éq. 8) & 19890 & & & \\
\hline & Production de pailles (éq. 8) & 1989 & & & \\
\hline & Produits nouveaux & 21879 & Perte d'usage de ressources & 0 & \\
\hline
\end{tabular}

* $1000 \mathrm{FCFA}=1,52 € ; * *$ éq. : équation (cf. Encadré 1)

\section{Tableau IX}

Bilan partiel de l'utilisation de fumier sur une culture de maïs

\begin{tabular}{|c|c|c|c|c|c|}
\hline & \multicolumn{2}{|c|}{$\begin{array}{l}\text { Economies des charges } \\
\text { ou génération de produits nouveaux (FCFA *) }\end{array}$} & \multicolumn{2}{|c|}{$\begin{array}{l}\text { Augmentation des charges } \\
\text { ou perte d'usage de ressources (FCFA) }\end{array}$} & \multirow[t]{4}{*}{$\begin{array}{l}\text { Bilan } \\
\text { (FCFA) }\end{array}$} \\
\hline \multirow{7}{*}{$\begin{array}{l}\text { Application } \\
\text { raisonnée } \\
\text { de fumure } \\
\text { organique }\end{array}$} & & & Transport FO (éq. 7) ** & 1666 & \\
\hline & & & Epandage (éq. 6) & 2085 & \\
\hline & & & Désherbage suppléé (éq. 6) & 855 & \\
\hline & Charges anciennes & 0 & Charges nouvelles & 4606 & \multirow[t]{4}{*}{31173} \\
\hline & Production de grains (éq. 8) & 32526 & & & \\
\hline & Production de pailles (éq. 8) & 3253 & & & \\
\hline & Produits nouveaux & 35779 & Perte d'usage de ressources & 0 & \\
\hline
\end{tabular}

* $1000 \mathrm{FCFA}=1,52 € ; * *$ éq. : équation (cf. Encadré 1$)$ 


\section{Bilan partiel de la construction des fosses à l'utilisation de la fumure organique (FO) au champ}

\section{Compost Fumier $($ FCFA *) (FCFA)}

$\begin{array}{lrr}\text { Pour une fosse de } \mathbf{9} \mathbf{~ m}^{\mathbf{3}} \\ \text { Augmentation de la capacité de production } & -2900 & -2900 \\ \text { Production de FO } & -8600 & -22450 \\ \text { Utilisation de FO } & 20081 & 31173 \\ \text { Bilan à l'échelle d'une fosse de } 9 \mathrm{~m}^{3} & 8581 & 5823\end{array}$

\section{Pour un hectare de maïs fumé}

[Nb. de fosses de $9 \mathrm{~m}^{3}$ nécessaires

$3,9 \quad 2,4]$

Augmentation de la capacité de production

Production de FO

$-11373-6954$

Utilisation de FO

$-33725-53837$

$78749 \quad 74750$

Bilan à l'échelle d'un hectare de maïs fumé

$33651 \quad 13964$

$* 1000 \mathrm{FCFA}=1,52 €$

L'amendement d'une parcelle avec du compost implique des investissements plus importants à réaliser pour construire le nombre de fosses nécessaires que pour un amendement avec du fumier. Il faut en effet 3,9 fosses à compost de $9 \mathrm{~m}^{3} / \mathrm{ha}$ et seulement 2,4 fosses fumières/ha.

Les coûts de travail de la production du compost sont élevés (8 600 FCFA ou 13,1 €) mais restent couverts par les gains de production en grain de maïs et en paille. Il n'y a pas de changement d'allocation des ressources ni de coût d'opportunité. Les coûts supplémentaires de production du fumier sont les travaux de transport de la fosse vers les champs (1 666 FCFA ou 2,5€) et la perte d'usage des ressources qui auraient pu être valorisées autrement (16 200 FCFA soit $24,7 €$ ).

Le gain de production obtenu par application de fumier sur un hectare de maïs correspond à 13924 FCFA $(21,2 €)$, ce qui équivaut à 0,9 sac d'engrais composés NPKSB. L'application de compost sur un hectare de maïs permet de dégager $33651 \operatorname{FCFA}(51,3 €)$, soit l'équivalent de 2,2 sacs d'engrais composés NPKSB.

Les deux modes de production de FO présentés ne sont pas antinomiques. Ils peuvent être développés dans la même exploitation si les travaux de construction, de remplissage et de vidange des fosses peuvent être assurés à des périodes différentes. Un modèle de deux fosses par exploitation, implantées aux champs et à la concession, peut être proposé aux producteurs.

\section{DISCUSSION}

La capacité de transport des exploitations reste une forte contrainte à la production de fumure organique (19) mais elle est réduite en plaçant les fosses à l'endroit où les biomasses sont disponibles. Cette étude montre également que le temps de travail (ramassage des résidus, des déjections, retournement des fosses, épandage de la FO) est un coût de production important de l'économie de production de FO et qu'il peut devenir une contrainte à la production lorsque le volume de FO augmente ou que la main d'œuvre dans l'exploitation est limitée.
Les bilans partiels fournissent une représentation des effets directs du processus innovant. Leur mise en débat avec les paysans a permis de discuter des facteurs de variations de ces bilans et d'identifier certaines opportunités pour les améliorer. Ainsi, les risques encourus par l'adoption de l'innovation sur la cohésion sociale (augmentation des charges en travail individuel vs renforcement de l'entraide, privatisation des résidus de culture $v s$ vaine pâture), l'accroissement attendu des revenus ou de la production céréalière ont pu être abordés avec les paysans. De même, les conditions nécessaires à la mise en place de l'innovation ont pu être précisées (possession d'animaux, disponibilité en main d'œuvre, appartenance à un groupe d'entraide, par exemple). Des freins à l'adoption de l'innovation ont pu être identifiés permettant de construire, selon la démarche de RAP, des propositions d'amélioration et ainsi de favoriser le changement de pratiques : entre autres, manque de disponibilité des outils corrigé par une gestion collective d'un stock d'outils, pénibilité du travail de creusement limitée par une organisation collective par groupement, dispersion des tiges de coton en fin de saison sèche limitée par une collecte précoce.

La méthode proposée permet de réaliser une évaluation globale des effets directs d'un processus innovant composé de plusieurs phases : la construction de fosse, la production et l'utilisation de FO. Cette méthode a l'avantage de prendre en compte l'ensemble des coûts liés à l'innovation au-delà de son impact sur les rendements des cultures ou la marge brute.

La méthode est applicable à tous les types de pratiques si le périmètre du système à évaluer et la situation de référence sont bien définis, et si l'inventaire des produits et des charges est élaboré. Cependant, la conversion en valeur monétaire des charges et des produits est souvent difficile (manque de références ou produits non marchands). Cette évaluation quantitative doit être complétée par une évaluation qualitative et participative pour nuancer les conclusions, prendre en compte les représentations des producteurs et les produits non marchands (fertilité des terres, qualité de la FO, pics de travail, etc.).

\section{- CONCLUSION ET PERSPECTIVES}

Cet article propose une méthode d'évaluation d'un processus innovant appliquée aux modèles de la fosse à compost et de la fosse fumière. Cette méthode se base sur l'analyse des effets directs, des conséquences indirectes et de la dynamique de l'innovation. Les effets directs sur l'économie de la FO dans les exploitations sont estimés à partir de la méthode du budget partiel pour chaque composante de l'innovation, enrichie par une caractérisation des sources de variation des termes du bilan par les producteurs. Les conséquences indirectes sur l'économie des exploitations et de ses interactions avec les systèmes de production et les filières, la société et l'environnement, ainsi que la dynamique locale de l'innovation doivent compléter cette analyse.

Les limites de la méthode du budget partiel résident dans la nécessité de convertir tous les produits et les charges en terme monétaire. Les méthodes de conversion ne sont pas toujours fiables et n'ont pas nécessairement de sens pour les paysans, particulièrement pour les produits non marchands. Cependant, la méthode permet de disposer d'une représentation simplifiée des effets directs de l'innovation pouvant intégrer une démarche de RAP et servir à la discussion avec les producteurs sur les modalités de l'innovation, les facteurs de variation des bilans, les propositions techniques ou organisationnelles efficientes et les mesures d'appui conseil à proposer. A partir de ce bilan, il est possible d'engager des échanges avec les producteurs sur l'analyse des conséquences indirectes et des enquêtes sur la dynamique locale de l'innovation. 


\section{BIBLIOGRAPHIE}

1. ALTER N., 2000. L'innovation ordinaire. Paris, France, PUF, 278 p.

2. BATIONO A., KIHARA J., VANLAUWE B., WASWA B., KIMETU J., 2007. Soil organic carbon dynamics, functions and management in West African agro-ecosystems. Agric. Syst., 94: 13-25.

3. BERGER M., 1996. L'amélioration de la fumure organique en Afrique Soudano-sahélienne. Agric. Dev. (hors-série) : 1-58.

4. BLANCHARD M., 2010. Gestion de la fertilité des sols et rôle du troupeau dans les systèmes coton-céréales-élevage au Mali-Sud : Savoirs techniques locaux et pratiques d'intégration agriculture élevage. Thèse Doct., Université Paris Est, Créteil, France, 301 p.

5. BLANCHARD M., VALL E., 2010. Production et utilisation de la fumure organique au Mali-Sud : savoirs des paysans face aux savoirs des agronomes, quels enseignements pour le développement ? In : Thibaud B., François A., éds., Systèmes de production et durabilité dans les pays du Sud. Paris, France, Karthala, p. 59-76.

6. BROSSIER J., CHIA E., MARSHALL E., PETIT M., 2003. Le budget partiel, cadre général de raisonnement de toute décision. In : Brossier J., Chia E., Marshall E., Petit M., éds., Gestion de l'exploitation agricole familiale, éléments théoriques et méthodologiques. Dijon, France, Enesad, Cnerta, p. 133-158.

7. CHIA E., 2004. Principes, méthodes de la recherche en partenariat: une proposition pour la traction animale. Rev. Elev. Méd. vét. Pays trop., $57: 233-240$.

8. DUGUE P., 1999. Utilisation de la biomasse végétale et de la fumure animale : impacts sur l'évolution de la fertilité des terres en zone de savanes. Etude de cas au Nord-Cameroun et essai de généralisation. Rapport final de l'ATP Flux de biomasse et gestion de la fertilité à l'échelle du terroir. Montpellier, France, Cirad-Tera, 175 p. (Rapport n 57-99)

9. DUGUE P., VALL E., LECOMTE P., KLEIN H.D., ROLLIN D., 2004. Evolution des relations entre l'agriculture et l'élevage dans les savanes d'Afrique de l'Ouest et du Centre : un nouveau cadre d'analyse pour améliorer les modes d'intervention et favoriser les processus d'innovation. Ol. Corps gras Lipides, 11 : 268-276.

10. GAFSI M., 2007. Gestion de I'exploitation agricole familiale africaine. In : Gafsi M., Dugue P., Jamin J-Y., Brossier J., 2007. Exploitations agricoles familiales en Afrique de l'Ouest et du Centre : enjeux, caractéristiques et éléments de gestion. Versailles, France, Quae, p. 211-301.

11. HALL A., DORAI K., KAMMLI T., 2012. Evaluating agricultural innovation system interventions. In: Agricultural innovation systems: An investment sourcebook. Washington DC, USA, World Bank, p. 580-588.
12. HAMON R., 1972. L'habitat des animaux et la production d'un fumier de qualité en zone tropicale. Agron. trop., 27 : 592-607.

13. HORTON D., MACKAY R., 2003. Using evaluation to enhance institutional learning and change: recent experiences with agricultural research and development. Agric Syst., 78: 127-142.

14. KOUTOU M., VALL E., 2010. Implication des acteurs locaux dans la conception d'innovations : le cas des systèmes agropastoraux du Tuy (Burkina Faso). In : Coudel E., Devautour H., Soulard C., Hubert B., eds., Int. Symp. Innovation and sustainable development in agriculture and food, Montpellier, France, 28 juin - 1 juil. 2010, 12 p.

15. LANDAIS E., LHOSTE P., 1990. L'association agriculture-élevage en Afrique intertropicale : un mythe techniciste confronté aux réalités de terrain. Cah. Sci. Hum., 26, 217-235.

16. MACKAY R., HORTON D., 2003. Expanding the use of impact assessment and evaluation in agricultural research and development. Agric. Syst., 78: 143-165.

17. MANLAY R.J., CHOTTE J.-L., MASSE D., LAURENT J.-Y., FELLER C., 2002. Carbon, nitrogen and phosphorus allocation in agro-ecosystems of a West African savanna. III. Plant and soil components under continuous cultivation. Agric. Ecosyst. Environ., 88: 249-269.

18. MUSTIN M., 1987. Le compost, gestion de la matière organique. Paris, France, François Dubusc, 954 p.

19. SCHLEICK K., 1986. Le fumier peut-il remplacer la jachère ? Possibilité d'utilisation du fumier : exemple de la savane d'Afrique occidentale. Rev. Elev. Méd. vét. Pays trop., 39 : 97-102.

20. VALL E., DUGUE P., BLANCHARD M., 2006. Le tissage des relations agriculture-élevage au fil du coton, 1990-2005. Cah. Agric., 15 : 72-79.

21. VALL E., KOUTOU M., BLANCHARD M., COULIBALY K., DIALLO M.A., ANDRIEU N., 2012. Intégration agriculture-élevage et intensification écologique dans les systèmes agrosylvopastoraux de I'Ouest du Burkina Faso, province du Tuy. In : Vall E., Andrieu N., Chia E., Nacro H.B., éds, Partenariat, modélisation, expérimentations : quelles leçons pour la conception de l'innovation et l'intensification écologique? Bobo-Dioulasso, Burkina Faso, 15-17 nov. 2011. http://hal. archives-ouvertes.fr/hal-00718613

22. VAN DER WERF H.M.G., TZILIVAKIS J., LEWIS K., BASSET-MENS C., 2007. Environmental impacts of farm scenarios according to five assessment methods. Agric. Ecosyt. Environ., 118: 327-338.

Mis en ligne en juin 2013 


\section{Summary}

Blanchard M., Koutou M., Vall E., Bognini S. How do we evaluate an innovative process? Case of the quantitative and qualitative improvement of organic manure in the field

Soil fertility in West Africa is under high anthropogenic pressure caused by the increasing population, extension of cultivated areas and abandonment of fallows. Through a participatory action research approach, the Fertipartenaires project proposed to design with farmers innovations on the use of organic manure, in three steps: increase the production capacity of organic manure by building pits, improve production quality, and integrate its application in the field. How do we assess an innovative process made up of several stages that cover different space and time scales? How do we include this assessment in a participatory approach? A participatory assessment method in three steps is proposed: (1) assessment of the direct effect of innovation (partial assessment), (2) analysis of economic, sociological and ecological indirect consequences of the innovation, and (3) analysis of innovation dynamics (adoption, dissemination, and redefinition). Two models of organic manure production have been tested: a manure pit built on the concession and a compost pit built at the edge of the field. The analysis of the direct effects of compost production shows that it is economically more advantageous than manure with its higher opportunity costs. However, the two production models are complementary and they each have a positive balance. The method used helps to assess the direct effects of innovation on the economic impact of organic manure production. It helps start discussions with farmers around the simplified illustration of the direct effects of innovation. It should however be completed by the analysis of indirect consequences and innovation dynamics so as to take into account the dimensions that were not included in the partial assessments.

Keywords: Soil fertility - Organic amendment - Fertilizer Innovation - Ex-post impact assessment - West Africa.

\section{Resumen}

Blanchard M., Koutou M., Vall E., Bognini S. Cómo evaluar un proceso innovador? Caso del mejoramiento cuantitativo y cualitativo del estiércol orgánico en el campo

La fertilidad de los suelos en África del oeste está sometida a fuertes presiones antropogénicas, con el aumento demográfico, la extensión de las superficies cultivadas y el abandono de la práctica de barbecho. Mediante la movilización de un proceso de investigación acción con colaboración, el proyecto "Fertipartenaires" propuso la co concepción de innovaciones sobre el uso del estiércol orgánico en tres etapas: aumentar la capacidad de producción de estiércol orgánico mediante la construcción de fosas, el mejoramiento de la calidad de producción y el uso racional de su aplicación en el campo. Cómo evaluar un proceso innovador compuesto de varias fases, e inscrito en escalas espaciales y temporales diferentes? Cómo incorporar esta evaluación en un proceso participativo? Se propuso un método participativo de evaluación en tres etapas: (1) evaluación del efecto directo de la innovación (recuento parcial), (2) análisis de las consecuencias indirectas económicas, sociológicas y ecológicas de la innovación y (3) análisis de la dinámica de la innovación (adopción, difusión y redefinición). Se probaron dos modelos de producción de estiércol: una fosa de estiércol instalada en concesión y una fosa de abono en la periferia del campo. El análisis de los efectos directos muestra que el uso del abono es económicamente más ventajoso que el del estiércol, con costos de oportunidades más elevados. Sin embargo, los dos modelos de producción son complementarios y presentan un balance monetario positivo. El método analizado permite reconocer los efectos directos de la innovación sobre la economía de producción de estiércol orgánico. Este permite lanzar un diálogo con los autores alrededor de la ilustración simplificada de los efectos directos de la innovación. Debe sin embargo completarse con un análisis de las consecuencias indirectas y de la dinámica de la innovación para considerar las dimensiones, del cual no toman cuenta los recuentos parciales.

Palabras clave: Fertilidad del suelo - Enmienda orgánica Abonos - Innovación - Evaluación del impacto ex post Africa Occidental. 